\title{
Stryker Osteonics: Prosthetic Knee Joint
}

\author{
P.G. HJORTH ${ }^{1} \dagger$ \\ ${ }^{1}$ Department of Applied Mathematics and Computer Science, Technical University of Denmark
}

(Communicated to MIIR on 27 July 2021)

Study Group: ESGI 62. 21-25/01/2008, Limerick, Ireland.

Communicated by: Sarah Mitchell

Industrial Partner: Stryker Osteonics

Presenter: C. Birkinshaw, University of Limerick; S. Flanagan, University of Limerick.

Team Members: P.G. Hjorth, Technical University of Denmark; J. King, University of Nottingham; A. Korobeinikov, University of Limerick; J. Mason, Bristol University; S. McKee, University of Strathclyde; S. Wilson, University of Strathclyde

Industrial Sector: Biomedical engineering, Materials, Prosthetics

Tools: Tribology, Contact mechanics, Poroelasticity

Key Words: Biomechanics, Porous media, Lubrication

MSC2020 Codes: 74, 92

\section{Summary}

This report studies the biomechanics, in particular thin-film lubrication, of an artificial knee joint. We study whether using a porous (but still stress resiliant) material, or simply adding to the current bearing design a large number of small drilled holes, makes it possible to entrap a lubricating fluid, in a way similar to what the living tissue does. The report concludes that such a design is indeed possible and may lead to less friction in low-load situation; but that the high-load situation is less clear. Also, the report considers only sliding movement of the bearing, not a combination of sliding and rolling.

$\dagger$ email: pghj@dtu.dk 


\section{The Problem}

The human knee is a hinge-type joint comprising two major bones, the femur and the tibia. The bone is end capped with a soft compliant substance cartilage, which acts as a low friction bearing. Lubrication is added by the cartilage synovial fluid, which ensures that the bearing works with full fluid film lubrication.

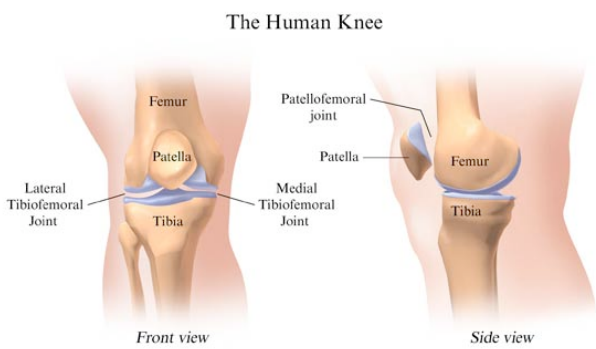

Figure 1: The human knee joint.

As the knee articulates, fluid is drawn through the joint space, ensuring that the two opposing cartilage surfaces do not come into contact. The load applied to the knee depends upon the activity, and can be as much as 8 times body weight. The cartilage also acts as a shock absorber, dampening the excessive loads applied and protecting the bones from shock. Synovial fluid is non-Newtonian, and when the joint is stationary, the fluid is almost gellike. The cartilage is porous, and never dries out in the healthy knee.
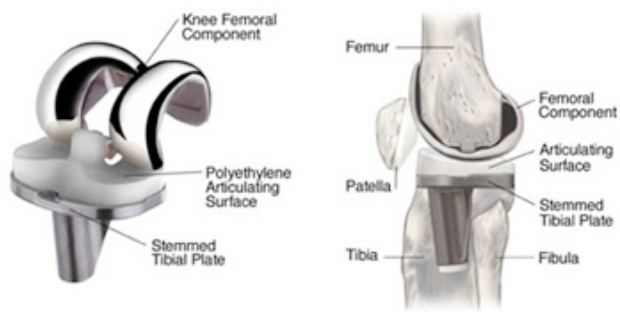

Figure 2: Elements of a prosthetic knee joint. The tibia is capped with a layer of polyurethane.

In the diseased knee cartilage breaks down, and the articulation tends to a bone-on-bone bearing. This is painful, and can be operated on to replace the diseased joint with a prosthetic joint. See figure 2 . 
The material properties of the prosthetic joint do not simulate cartilage in general. However, a new type of material is being considered polyurethane (PU), which simulates cartilage more closely than the conventional materials used in joint replacement. The system being considered is a PU tibial bearing articulating against a metal femoral bearing.

The PU is not porous, but can develop full fluid film lubrication if the conditions are correct. Many mathematical approaches ([2]-[11]) have been adopted to explore the nature and tribology of lubrication between poroelastic surfaces. The entire lubrication process is complex and involves the simultaneous solution of the equations of fluid dynamics with the equations of Hertian contact mechanics.

In the joints manufactured, it is necessary to have a soft, compliant PU as the bearing material. Under load, this material is easily deformable, and as the load increases so the contact area increases, thereby lowering the contact stress. The soft PU bearing is supported by a harder PU backing. The interface between the two materials is formed by fusion bonding, and is consequently an inherent weakness in the system. The weakness is most noticeable at the edges; when the material is loaded and the soft PU deforms, the displaced material exerts a shear force at the interface.

The Study Group was asked

(1) To investigate the inclusion of holes / channels in the loaded area of bearing to determine whether these features can: entrap fluid and thus simulate a porous surface, and can release fluid when the bearing is articulated to maximise the development of a fluid film.

(2) To investigate if holes around the periphery of the bearing can reduce the shear stress at the interface.

\section{Thin Film Lubrication Theory}

In thin film lubrication theory (see e.g., [1]), one considers a 2-D model of two surfaces in relative motion, and a thin layer of fluid in between.

Let $U_{R}$ be the relative velocity of the two surfaces (here given to the upper surface), and $h(x)$ the $x$-dependent separation of the surfaces. See figure 3. 


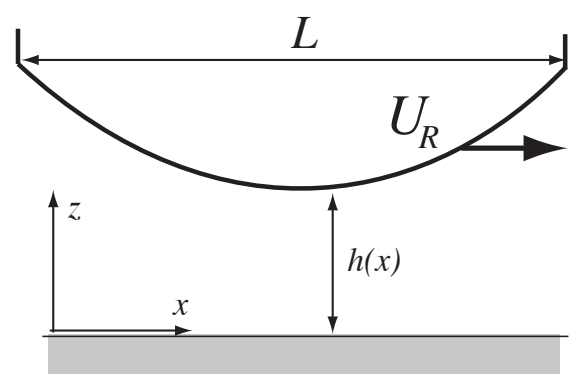

Figure 3: Thin film model. The fluid forms a thin layer of width $h(x)$ between the femur (top) and the tibia (bottom) surfaces. The curvature relative to other distances is exaggerated. The top surface moves with a velocity $U_{R}$.

The horizontal velocity field in the film is approximatly given ([1]) by

$$
u(x, z)=\left[\frac{U_{R}}{h}-\frac{z}{2 \mu} p_{x}\right](h-z)
$$

where $\mu$ is the fluid viscosity, and $p_{x}$ is the $x$-derivative of the pressure $p$.

The overpressure $p-p_{0}$ (which in the joint supports the vertical load) is connected to the width function $h(x)$, by:

$$
\frac{p-p_{0}}{6 \mu}=U_{R} \int^{x} \frac{1}{h^{2}(s)} \mathrm{d} s-2 Q \int^{x} \frac{1}{h^{3}(s)} \mathrm{d} s
$$

where $U_{R}$ is the relative velocity, and $Q$ is the volume flux related to $U_{R}$ by:

$$
Q=\int_{0}^{h(z)} u \mathrm{~d} z=\frac{1}{2} U_{R} h-\frac{h^{3}}{12 \mu} p_{x}
$$

\section{A Surface cavity}

Even from the lowest order equations (2) and (3) one can estimate the effect of changes in the height function $h(x)$. Suppose a small hole is drilled into the PU tibia surface, as in figure $4(b)$.

The small hole will be filled with fluid, since the large pressure forces in the joint are much greater than the subtle effects like capillary forces. 

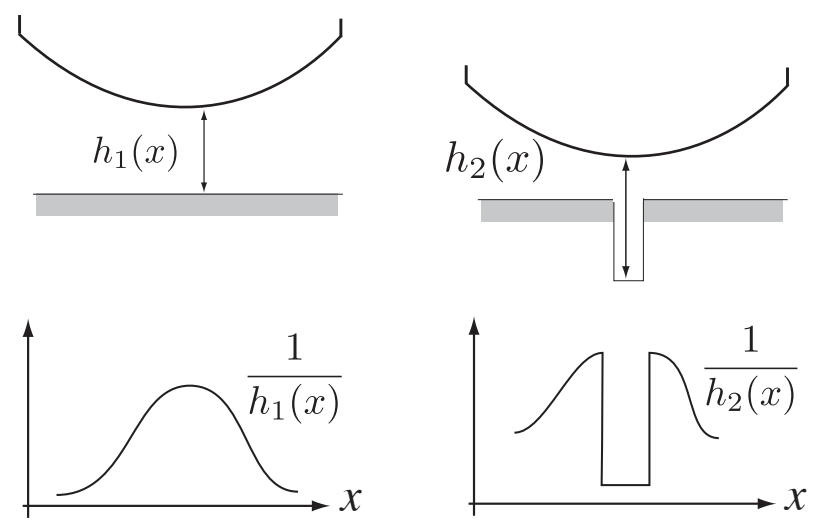

Figure 4: Two distance functions, $h_{1}$ and $h_{2}$, compared.

The hole causes a new height function $h_{2}(x)$ of the fluid. The pressure will, for a fixed contact surface area, always ajust itself to the constant body weight and thus be a constant. Since the hole introduces a local increase in $h$, the new $h_{2}(x)$ will have lower values in the region where there is no hole, keeping the integral over the $1 / h^{2}(x)$ terms in the pressure integrals equation (2) constant.

Since thin-film theory predicts that the pressure is of order $\mu U L / h^{2}$ while the tangential stresses are of order $\mu U / h$, horizontal forces in this model are smaller than vertical forces by a factor of order $h / L$.

Thus, a hole (and even more a collection of small holes) will lead to a smaller minimal value of $h(x)$. A smaller $h$ means less tangential stress, in other words less friction in the bearing. On the other hand, a too small value of $h$ brings the hard surfaces even closer to undesired contact.

\section{Poroelasticy}

A number of studies of the properties of poroelastic systems like the knee joint has been performed; see e.g., recent works [10] and [11], where a simplified model of joint articulation in the presence of both a non-Newtonian synovial fluid and poroelastic surfaces have been studied. 
Even though a PU surface is not porous, it is interesting to compare the conclusion of the previous section with results from more complex calculations involving poroelastic surfaces with varying degrees of roughness.

We therefore briefly outline, following [11], an extension of the model to a poroelastic system, albeit without the feature of a couple-stress synovial fluid. The properties of such a fluid seems nevertheless relevant for the system and should be considered in further studies.

In figure 5, the tibia base is now poroelastic, meaning that the material has elastic properties as well as the ability to entrain fluid in a microscopic structure of canals, so that one should consider both a material displacement field $\mathbf{U}$, and the average fluid field $\mathbf{V}$ inside the poroelastic in addition to the 'real' fluid field $(u, v)$ outside the poroelastic.

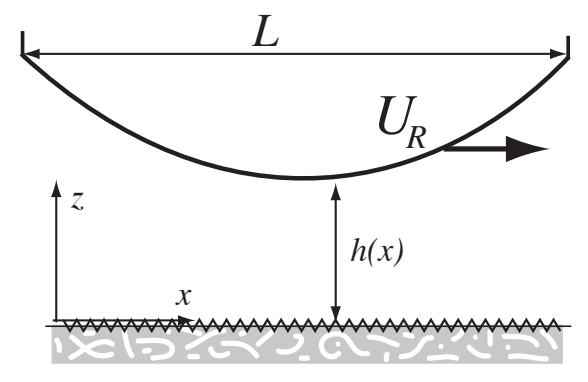

Figure 5: A poroelastic problem.

The distance function $h$ now consists of two terms, $h(x)=h_{0}(x)+h_{s}$, where $h_{s}$ describes a random variable with zero mean and a deviation which characterizes the roughness of the surface.

Using a statistical average, and scaling with the natural length scale $L$, one can obtain a dimensionless quantity $C$ which is a measure of the relative roughness of the poroelastic surface.

The fluid field is considered to be incompresible:

$$
\frac{\partial u}{\partial x}+\frac{\partial v}{\partial y}=0
$$

as well as satisfying

$$
\mu \frac{\partial^{2} u}{\partial y^{2}}=\frac{\partial p}{\partial x} \quad \text { and for the presure } p \quad \frac{\partial p}{\partial y}=0
$$


In the poroelastic region, the material displacement field $\mathbf{U}$

$$
\rho_{m} \frac{\partial^{2} \mathbf{U}}{\partial t^{2}}=\operatorname{div} \sigma_{m}-\frac{\mu}{k}\left(\frac{\partial \mathbf{U}}{\partial t}-\mathrm{V}\right)
$$

In the fluid region, we $\mathbf{U}$, Fluid field $\mathbf{V}$

$$
\rho_{f} \frac{\partial^{2} \mathbf{V}}{\partial t^{2}}=\operatorname{div} \sigma_{f}+\frac{\mu}{k}\left(\frac{\partial \mathbf{U}}{\partial t}-\mathrm{V}\right)
$$

where $\sigma_{m}$ and $\sigma_{f}$ are stress tensors. The pressure $P$ in the pores will satisfiy

$$
\nabla^{2} P=0
$$

Boundary conditions for the velocity field will be that at $z=0$ :

$$
u=0, \quad v=-v_{n}, \quad \frac{\partial^{2} u}{\partial z^{2}}=0
$$

At $z=h_{0}$ :

$$
u=0, \quad v=-v_{n}-\frac{\mathrm{d} h_{0}}{\mathrm{~d} t}, \quad \frac{\partial^{2} u}{\partial z^{2}}=0
$$

In [11], it is found that such a system has a spatial pressure distribution which depends on the roughness parameter $C$. See figure 6 .

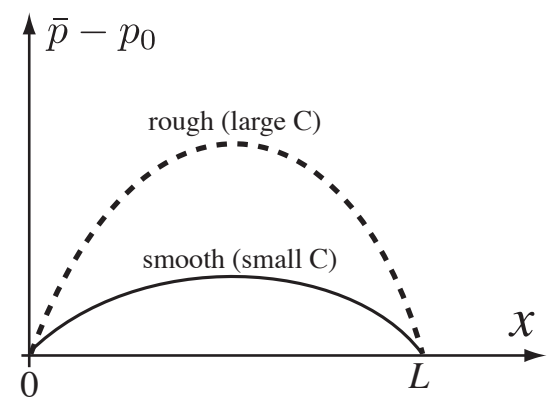

Figure 6: Dimensionless overpressure $\bar{p}-p_{0}$ across the joint for small and large values of the dimensionless roughness parameter $C$.

A load-carrying capacity may be defines as

$$
\bar{W}=\int_{0}^{L} \bar{p}-p_{0} \mathrm{~d} x
$$




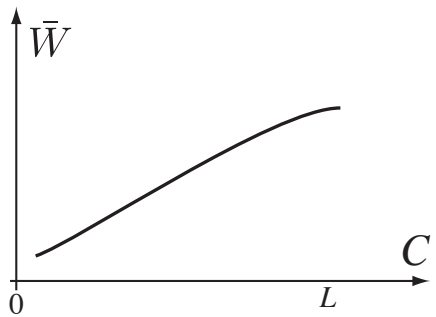

Figure 7: Dimensionless load $W$ capacity as a function of the dimensionless roughness parameter $C$.

It is established in [11] that the load capacity is an increasing function of the roughness parameter $C$. It is established in [11] that the load capacity is an increasing function of the roughness parameter $C$.

\section{Conclusion}

We have examined, within a simple bearing model of a knee joint, the effect on the fluid film properties of the presence of a small vertical hole in the load area. The calculations indicates that

Fluid is entrapped in such a hole (in a porous medium, fluid can also be released, and may thus be 'pumped' in and out, so the porous layer can act as a reservoir of fluid).

In a simple model, the presence of the hole will, for constant load, cause a smaller minimal film separation of the two surfaces. This will lower the horizontal friction, but may also bring about surface contact in high load situations.

We note that this result, that small holes gives better lubrication is consistent with [11] and also with recent results examining both slide and roll [12].

The model is incomplete in that it consideres pure sliding, not the combined effect of sliding and rolling, and also does not consider the elastic deformation of the PU surface. Also, we did not consider the second question of the effect of holes to reduce sheer stresses at the soft PU - hard PU interface. 


\section{References}

[1] A.C. Fowler: Mathematical Models in the Applied Sciences, Cambridge Texts in Applied Mathematics (1995).

[2] P.A. Torzilli, V.C. Mow: On the fundamental fluid transport mechanics through normal pathological articular cartilage during function, J. Biomech. 9, 587, (1995).

[3] L. Blankevoort, J.H. Kuiper, R. Huiskes, H.J. Grootenboer: Articular contact in a three-dimensional model of the knee, J. Biomech. 24, 1019, (1991).

[4] J.S. Hou, V.C. Mow, W.M. Lai, M.H. Holmes: An analysis of the squeeze-lm lubrication mechanism for articular cartilage, J. Biomech. 25, 247, (1992).

[5] J.R. Sachs, M.R. Glucksberg, O.E. Jensen, J.B. Grotberg: Linear ow and deformation in a poroelastic disk with a free surface, Trans. ASME J. Appl. Mech, 61, 726, (1994).

[6] O.E. Jensen, M.R. Glucksberg, J.R. Sachs, J.B. Grotberg: Weakly Nonlinear deformation of a thin poroelastic layer with a free surface, Trans. ASME J. Appl. Mech. 61, 729, (1994).

[7] V.K. Stokes: Couple stresses in fluids, Phys. Fluids 9, 1709, (1966).

[8] S.I. Barry, M. Holmes: Asymptotic behaviour of thin poroelastic layers, J. Biomech. 66, 175, (2001).

[9] H. Christensen: Stochastic model for hydrodynamic lubrication of rough surfaces, Proc. Inst. Mech. Eng. 184, 1013, (1969).

[10] N.M. Bujurke, R.B. Kudenatti: Surface roughness effects on squeeze lm poroelastic bearings Applied Mathematics and Computation 174, 1181, (2006).

[11] N.M. Bujurke, R.B. Kudenatti: An analysis of rough poroelastic bearings with reference to lubrication mechanism of synovial joints Applied Mathematics and Computation 178, 309, (2006).

[12] I. Krupka, M. Hartl: Thin-film Lubrication of Dented Surfaces Tribology Transcations (STLE Annual Meeting, 2007) ISSN 1040-2004, 488, (2007). 